\title{
A multicentre matched case control study of risk factors for Preeclampsia in healthy women in Pakistan
}

\author{
Uzma Shamsi*1, Juanita Hatcher' ${ }^{1}$, Azra Shamsi2, Nadeem Zuberi3 , Zeeshan Qadri1 and Sarah Saleem¹
}

\begin{abstract}
Background: Preeclampsia is one of the leading causes of maternal and perinatal morbidity and mortality world-wide. The risk for developing preeclampsia varies depending on the underlying mechanism. Because the disorder is heterogeneous, the pathogenesis can differ in women with various risk factors. Understanding these mechanisms of disease responsible for preeclampsia as well as risk assessment is still a major challenge. The aim of this study was to determine the risk factors associated with preeclampsia, in healthy women in maternity hospitals of Karachi and Rawalpindi.

Methods: We conducted a hospital based matched case-control study to assess the factors associated with preeclampsia in Karachi and Rawalpindi, from January 2006 to December 2007. 131 hospital-reported cases of PE and 262 controls without history of preeclampsia were enrolled within 3 days of delivery. Cases and controls were matched on the hospital, day of delivery and parity. Potential risk factors for preeclampsia were ascertained during in-person postpartum interviews using a structured questionnaire and by medical record abstraction. Conditional logistic regression was used to estimate matched odds ratios (ORs) and 95\% confidence intervals ( $95 \% \mathrm{Cls}$ ).

Results: In multivariate analysis, women having a family history of hypertension (adjusted OR 2.06, 95\% Cl; 1.27-3.35), gestational diabetes (adjusted OR 6.57, 95\% Cl; 1.94 -22.25), pre-gestational diabetes (adjusted OR 7.36, 95\% Cl; 1.3733.66) and mental stress during pregnancy (adjusted OR 1.32; 95\% Cl; 1.19-1.46, for each 5 unit increase in Perceived stress scale score) were at increased risk of preeclampsia. However, high body mass index, maternal age, urinary tract infection, use of condoms prior to index pregnancy and sociodemographic factors were not associated with higher risk of having preeclampsia.

Conclusions: Development of preeclampsia was associated with gestational diabetes, pregestational diabetes, family history of hypertension and mental stress during pregnancy. These factors can be used as a screening tool for preeclampsia prediction. Identification of the above mentioned predictors would enhance the ability to diagnose and monitor women likely to develop preeclampsia before the onset of disease for timely interventions and better maternal and fetal outcomes.
\end{abstract}

\section{Background}

Preeclampsia (PE) is a pregnancy-specific condition and is associated with high maternal mortality and morbidity as well as risk of perinatal death, preterm birth, and intrauterine growth restriction[1]. It occurs in 4 to 7 per cent of pregnant women worldwide [2]. The etiology of preeclampsia remains unclear despite extensive research. Risk

* Correspondence: uzma.shamsi@aku.edu

1 Division of Epidemiology/Biostatistics, Department of Community Health Sciences, Aga Khan University, Karachi, Pakistan

Full list of author information is available at the end of the article factors for preeclampsia include nulliparity, a family or own history of PE, pre-existing diabetes or increased body mass index, multiple pregnancy, maternal age, renal disease, hypertension or raised blood pressure at booking and chronic autoimmune disease [3]. The rate of preeclampsia has increased worldwide especially in developed countries by $40 \%$ between 1990 and 1999 due to an increase in number of older mothers and multiple births, conditions known to increase its risk[4]. 
Maternal Mortality is extremely high in Pakistan where 1 in 89 women dies of maternal causes with preeclampsia and eclampsia as one of the major causes [5]. As preeclampsia remains a serious and poorly understood complication of pregnancy, we need to identify epidemiological and clinical risk factors to predict it before it threatens the survival of both mother and fetus. The study of risk factors and the underlying evidence base can be used to assess risk of preeclampsia at antenatal booking [6]. There is paucity in studies on preeclampsia and its associated factors in Pakistan; an economically developing country in Asia.

This study was conducted to determine factors associated with PE, in healthy women with single pregnancy in maternity hospitals of Karachi and Rawalpindi. Moreover, this study investigated the association between family history of hypertension and preeclampsia.

\section{Methods}

This matched case control study was conducted between January 2006-December 2007 in six selected maternity hospitals in Karachi and two maternity hospitals in Rawalpindi: Aga Khan University Hospital, Sohbraj Maternity Hospital, Aga Khan Maternity hospital for women, Karimabad, Aga Khan Maternity hospital for Women \& Children, Garden, Lady Dufferin Hospital and Jinnah Postgraduate Medical Centre Hospital in Karachi, and Combined Military Hospital and Military Hospital in Rawalpindi.

All pregnant women of any age delivering in any of the above-mentioned hospitals were potential study subjects. We excluded women with past histories of chronic hypertension, and multiple gestations in current pregnancies.

A case was defined as a woman who had given birth during the preceding three days and who, in the antenatal period or before going into labor, was diagnosed by an obstetrician as being preeclamptic. Preeclampsia was defined as pregnancy-induced hypertension associated with proteinuria. Pregnancy-induced hypertension was defined as new hypertension with blood pressure of 140 $\mathrm{mm} \mathrm{Hg}$ systolic or diastolic B.P of $90 \mathrm{~mm} \mathrm{Hg}$ diastolic or greater arising after 20 weeks of gestation in a woman who was normotensive before 20 weeks gestation. Proteinuria was defined as excretion of $300 \mathrm{mg}$ or more of protein in 24 hour urine sample or $1+$ or more on dipstick (ICD-9 codes 642E-Fand ICD - 10 code O15).

A control was defined as a woman who gave birth during the preceding 3 days and who did not have a diagnosis of preeclampsia. For each case we interviewed two controls who delivered on the same day in the same hospital matched on parity. In total 131 cases and 232 controls were interviewed. The hospital's register was surveyed each day by the study team members to identify all preeclamptic cases, and potential controls. Prior to the inter- view, informed consent was obtained from all study participants. Women with preexisting chronic hypertension, defined as BP greater than 140/90 $\mathrm{mmHg}$ before pregnancy or before 20 weeks' gestation, were excluded.

The data collection was conducted on the post delivery ward by the specialized doctors using a structured questionnaire. Women with singleton gestations delivered after 20 weeks were eligible for inclusion. They were interviewed at enrollment and within 3 days of delivery. The questionnaire included information regarding sociodemographic characteristics, antenatal history and family history of hypertension and diabetes apart from other covariates.

Potential risk factors were selected on the basis of literature review and biological plausibility for an association with both the exposure and outcome. The covariates included in the analysis were maternal age, maternal smoking, gestational diabetes, diabetes mellitus, stress measured by Perceived Stress Score PSS, sociodemographic status, age at menarche, body mass index, urine tract infections, past history of PE, family history of hypertension, diabetes mellitus, use of condoms, age of husband, sex of the baby, blood group and RH factor. Diabetes mellitus and gestational diabetes were self reported by the participants and verified by the medical records. Perceived Stress Scale (PSS) used was a 10-item measure of stressful situations during the past month [7]. Items are scored on a five-point scale from 0 to 4 ; the total score provides a global measurement of the extent to which an individual feels overwhelmed. Total scores range from 0 to 40; higher scores indicate greater perceived stress. As we don't have the data on incidence or prevalence of PE in Pakistan therefore the prevalence of various risk factors for pre-eclampsia was ascertained through literature search. In the sample size calculation, we assumed the prevalence of the various risk factors amongst the control group to be in the range of $11-72 \%$. The prevalence of Diabetes in Pakistan is 11\%. With low prevalence of disease as well as time and financial constraints, we took the option of taking a 1:2 ratio between cases and controls to increase the power of the study. Thence in order to be able to detect an odds ratio of at least 2 with a power of $80 \%$, at a significance level of $5 \%$, we calculated a sample size of 187 cases and 374 controls. \{NCSS statistical software \& power analysis sample size PASS $\}$.

All data analyses were performed using SAS, version 9.1, for windows (SAS Institute Inc. North Carolina) and SPSS 16. To identify the factors associated with PE, univariate matched analysis was done by comparing the cases and controls ( 1 case: 2 controls) for each variable of interest and crude matched odds ratio and their $95 \%$ confidence intervals along with $\mathrm{p}$ values were calculated. In multivariable analysis, matched analysis in logistic regression was performed to identify associated factors of 
PE while adjusting for other variables. Finally any variable with p-value $>0.05$, not a confounder or interacting with other variables were removed from the model to obtain a parsimonious and biologically meaningful model that best explains the phenomena of PE.

The study was approved by the Ethical Review Board of Aga Khan University Hospital.

\section{Results}

The sociodemographic, obstetric and antenatal characteristics of all participants are presented in table 1 . The actual sample size achieved was a total of 131 cases matched on parity, day of delivery and hospital with 262 controls participated in the study of preeclampsia risk factors. There were 194 nulliparous women and 199 parous women with single pregnancy. No significant differences were observed between cases and controls with regard to maternal age, age at menarche, maternal education, socioeconomic status measured by ownership of house, number of rooms in household, number of household members, husband's occupation.

Gestational diabetes was higher among cases $(12.4 \%)$ as compared to controls (1.9\%). Urine tract infections during pregnancy were higher among cases compared to controls (31\% among cases and $18.5 \%$ among controls). Pregestational diabetes was higher among cases (5.6\%) compared to controls $(0.8 \%)$. Family history of diabetes among first relatives was $43.8 \%$ among cases and $29.6 \%$ among controls. Family history of hypertension among cases was $58.9 \%$ as compared to $38.5 \%$ among controls. Mean Perceived stress score was 22 among cases as compared to 20 among controls. The mean prepregnancy weight for cases was $57.3 \mathrm{~kg}$ and for controls it was 55 kgs. There were no differences among cases and controls with regard to age of husbands, condom use around conception, sex of baby, maternal height, BMI, Blood group and $\mathrm{Rh}$ factor.

Referring to table 2, the significant variables in the final model were history of gestational diabetes, pregestational diabetes mellitus, family history of hypertension and perceived stress score. After adjusting for the effect of other variables in the model it was found that gestational diabetes was independently associated with preeclampsia. Women with gestational diabetes were at greater odds of having preeclampsia as compared to those who had no gestational diabetes $(\mathrm{OR}=6.57, \mathrm{CI} ; 1.94-22.25)$.

Similarly history of pre-gestational diabetes was independently associated with preeclampsia. Women with a history of pre-gestational diabetes were at greater odds of having preeclampsia as compared to women without pregestational diabetes $(\mathrm{OR}=7.36, \mathrm{CI} ; 1.37-33.66)$.

Our study showed that the family history of hypertension was an important risk factor for preeclampsia. Cases were 2.3 times more likely to have a positive family his- tory of hypertension as compared to controls, while keeping no family history of hypertension as the reference category $(\mathrm{OR}=2.06, \mathrm{CI} ; 1.27-3.35)$.

Mental stress during pregnancy, measured by Perceived Stress Scale, was also associated with increased risk of PE $(\mathrm{OR}=1.32$; CI; 1.19-1.46). For each 5 unit increase in the stress score the risk of preeclampsia increased by 1.32 . There was no collinearity between variables included in the final model.

\section{Discussion}

This is the first report of a hospital based case control study to determine preeclampsia risk factors among women in Karachi and Rawalpindi. Participants provided information on all the potential risk factors during postpartum interviews.

Our results showed that gestational diabetes mellitus (GDM) was associated with the subsequent development of preeclampsia. The result of our study showing a relationship between preeclampsia and diabetes is also consistent with previous findings [6,8-13]. Gestational diabetes is independently and significantly associated with an increased risk of preeclampsia and an even minor degree of glucose intolerance is associated with preeclampsia [14-16]. Similarly there was a positive association between pregestational diabetes and preeclampsia risk. This finding is also consistently seen in previous studies [15,17-20]. This association is based on a small number of cases and control subjects and the confidence interval is wide.

The findings from our study are biologically plausible for reason that epidemiological and clinical data document a close association between insulin resistance, type 2 diabetes, and hypertension. Hyperinsulinemia has been shown to stimulate the proliferation of vascular smooth muscle cells, enhance acute sympathetic nervous system activity and modify transmembrane cation transport, as well as renal sodium retention and associated endothelial dysfunction. All of these alterations may contribute to blood pressure elevations [21,22].

Our observation of a positive association between family history of chronic hypertension and risk of preeclampsia is consistent with several previous reports [23-25]. These studies reported an increase in risk of preeclampsia with positive family history of chronic hypertension. Our results suggest that family history of hypertension reflects genetic and behavioral factors whereby women may be predisposed to an increased preeclampsia risk. Family history of chronic hypertension is a proxy measure for hereditary factors as well as common environmental or behavioral exposures that may underlie preeclampsia risk $[26,27]$. To the best of our knowledge, this is the first analysis in Pakistan to assess the effects of 
Table 1: Table showing demographic, socio-economic and obstetrical characteristics of cases and controls with preeclampsia (PE) among women in maternity hospitals of Karachi and Rawalpindi (January 2006-December 2007)

\begin{tabular}{|c|c|c|c|}
\hline Variables & $\begin{array}{l}\text { Cases (131) } \\
\text { n (\%) }\end{array}$ & $\begin{array}{l}\text { Controls (262) } \\
\text { n (\%) }\end{array}$ & P-value \\
\hline \multicolumn{4}{|l|}{ Parity } \\
\hline Nulliparous women & 64 (48.9) & $130(49.6)$ & 0.915 \\
\hline Parous women & $67(51.1)$ & $132(50.4)$ & \\
\hline \multicolumn{4}{|l|}{ Mean maternal age (years) } \\
\hline$\leq 18$ & $5(3.8)$ & $12(4.6)$ & 0.935 \\
\hline $19-34$ & $114(87.0)$ & $226(86.6)$ & \\
\hline$\geq 35$ & $12(9.2)$ & $23(8.8)$ & \\
\hline Age at menarche & $13.0 \pm 1.2$ & $13.0 \pm 1.3$ & 0.476 \\
\hline Maternal Smoking & $5(3.8)$ & $16(6.1)$ & 0.42 \\
\hline \multicolumn{4}{|l|}{ Maternal education } \\
\hline Less than 8 years & $41(31.3)$ & $69(27.2)$ & 0.696 \\
\hline 8-12years & $64(48.9)$ & $131(51.6)$ & \\
\hline More than 12 years & $26(19.8)$ & $54(21.3)$ & \\
\hline \multicolumn{4}{|l|}{ Ownership Status } \\
\hline Owned & $91(69.5)$ & $160(61.1)$ & 0.102 \\
\hline Rented & $40(30.5)$ & $102(38.9)$ & \\
\hline \multicolumn{4}{|l|}{$\begin{array}{l}\text { Number of rooms in } \\
\text { household }\end{array}$} \\
\hline$\leq 3$ & $73(55.7)$ & $145(55.3)$ & 0.943 \\
\hline$>3$ & $58(44.3)$ & $117(44.7)$ & \\
\hline \multicolumn{4}{|c|}{$\begin{array}{l}\text { Mean number of household } \\
\text { members }\end{array}$} \\
\hline$\leq 6$ & $63(48.1)$ & $147(56.1)$ & 0.133 \\
\hline$>6$ & $68(51.9)$ & $115(43.9)$ & \\
\hline \multicolumn{4}{|l|}{ Husband's occupation } \\
\hline Private job & $42(32.1)$ & $98(37.4)$ & 0.296 \\
\hline Government job & $50(38.2)$ & $108(41.2)$ & \\
\hline Business & $29(22.1)$ & $44(16.8)$ & \\
\hline Others & $10(7.6)$ & $12(4.6)$ & \\
\hline \multicolumn{4}{|l|}{ Monthly household income } \\
\hline$>10000$ & $45(34.4)$ & $118(45.2)$ & 0.034 \\
\hline $4001-10000$ & $65(49.6)$ & $120(46.0)$ & \\
\hline$\leq 4000$ & $21(16.0)$ & $23(8.8)$ & \\
\hline Gestational diabetes & $16(12.4)$ & $05(1.9)$ & $<0.001$ \\
\hline Urinary tract infection & $39(31.0)$ & $47(18.5)$ & 0.006 \\
\hline $\begin{array}{l}\text { Pregestational Diabetes } \\
\text { Mellitus }\end{array}$ & $07(5.6)$ & $02(0.8)$ & 0.004 \\
\hline Family History of DM & $56(43.8)$ & $75(29.6)$ & 0.006 \\
\hline $\begin{array}{l}\text { Family History of } \\
\text { Hypertension }\end{array}$ & $76(58.9)$ & $100(38.5)$ & $<0.001$ \\
\hline Perceived Stress Score* & $22 \pm 6.1$ & $20 \pm 6.2$ & 0.002 \\
\hline Husband's Age* & $33.5 \pm 12.9$ & $33.5 \pm 13.5$ & 0.981 \\
\hline
\end{tabular}


Table 1: Table showing demographic, socio-economic and obstetrical characteristics of cases and controls with preeclampsia (PE) among women in maternity hospitals of Karachi and Rawalpindi (January 2006-December 2007)

\begin{tabular}{|c|c|c|c|}
\hline $\begin{array}{l}\text { Condom use around the } \\
\text { time of conception }\end{array}$ & $17(13.5)$ & $34(13.7)$ & 0.954 \\
\hline Height $^{*}$ (centimeters) & $150.8 \pm 22.3$ & $149.5 \pm 23.5$ & 0.592 \\
\hline Weight*(kilograms) & $57.3 \pm 15.8$ & $55.0 \pm 12.2$ & 0.002 \\
\hline \multicolumn{4}{|l|}{ BMI‡ } \\
\hline$<18.5$ & 18 (13.7) & $37(14.1)$ & 0.502 \\
\hline $18.5-24.9$ & 78 (59.5) & $171(65.3)$ & \\
\hline $25-29.9$ & $23(17.6)$ & $39(14.9)$ & \\
\hline$\geq 30$ & $12(9.2)$ & $15(5.7)$ & \\
\hline Gestational age ${ }^{*}$ (weeks) & $36.2 \pm 2.2$ & $37.0 \pm 1.2$ & $<0.001$ \\
\hline \multicolumn{4}{|l|}{ Blood Group } \\
\hline$A+/ A-$ & $30(24)$ & $47(18.4)$ & 0.326 \\
\hline $\mathrm{B}+/ \mathrm{B}-$ & $42(33.6)$ & $75(29.4)$ & \\
\hline$A B+/ A B-$ & $11(8.8)$ & $30(11.8)$ & \\
\hline $\mathrm{O}+\mathrm{OO}-$ & $42(33.6)$ & $103(40.4)$ & \\
\hline Rh Factor positive & $120(96)$ & $231(90.6)$ & 0.067 \\
\hline Rh factor negative & $5(4)$ & $24(9.4)$ & \\
\hline \multicolumn{4}{|l|}{ Sex of Baby } \\
\hline Male & $77(61.1)$ & $151(58.5)$ & 0.628 \\
\hline Female & 49 (38.9) & $107(41.5)$ & \\
\hline
\end{tabular}

*Plus-minus values are means \pm SD.

$\ddagger \mathrm{BMI}=$ body mass index, calculated by dividing weight in kilograms with height in meters squared.

family history of chronic hypertension on preeclampsia risk.

Women's family history of chronic hypertension is an important and easy to acquire clinical risk marker of preeclampsia compared to the biochemical markers. During the past 100 years numerous clinical, biophysical, and biochemical tests have been suggested to identify women who are at increased risk for future development of preeclampsia. Unfortunately, these biomarkers have limited sensitivity and are expensive enough for our women to be clinically useful for the prediction of preeclampsia in our setting. The family history of hypertension questions can be used as an inexpensive and feasible screening tool to identify pregnant women in a developing country like Pakistan to monitor the signs of preeclampsia during early pregnancy.

Our study showed an association of mental stress during pregnancy and development of preeclampsia. Depression and anxiety in early pregnancy are associated with risk for preeclampsia, a risk further increased if associated with vaginosis [28] Stressful work environment and stressful home environment are also associated with preeclampsia [29]. The prenatal stress alters maternal physiology and immune function in a manner consistent with increased risk of pregnancy complications such as preeclampsia and premature labor [30]. In another study work-related psychosocial strain increased the risk of PE [31]. Vasoconstriction in preeclampsia may develop early in pregnancy. Indeed, increased uterine artery resistance in maternal anxiety could be a primary manifestation or even the cause of preeclampsia [32,33].

Several limitations must be considered when interpreting the results from our study. First of all recall bias and inability to establish temporality between preeclampsia and certain variables are inherent due to the case control study design. We cannot exclude the possibility that our results could be partially confounded by unidentified risk factors. Our study would have been strengthened by a larger number of preeclamptic women, particularly those with histories of exposures like extremes of age, condom use, women with change in paternity. Prepregnancy weights and body mass index (BMI) were assessed by subtracting the average weight gain from the full term maternal weight which might not be a good proxy measure. There is certainly underreporting of smoking, condom use and therefore we could not establish a relationship between them and preeclampsia risk. Dietary and other lifestyle changes relationship with preeclampsia not analyzed in this study. This being a hospital based study; the results may not be applicable to the general population at large. We could not establish a relationship between certain factors like condom use and 
Table 2: Table based on multivariable analysis showing the association of independent variables with PE risk among women in maternity hospitals of Karachi and Rawalpindi (January 2006-December 2007)

\begin{tabular}{|c|c|c|}
\hline Variables & Matched unadjusted OR(95\% Cl) & Matched adjusted OR(95\%CI) \\
\hline Gestational diabetes & $6.40(2.34,17.4)$ & $6.57(1.94,22.25)$ \\
\hline Urinary tract infection & $2.0(1.21,3.49)$ & NS \\
\hline Pregestational Diabetes Mellitus & $6.56(1.36,31.7)$ & $7.36(1.37,33.66)$ \\
\hline Family History of DM & $1.88(1.18,3.00)$ & NS \\
\hline Family History of Hypertension & $2.24(1.45,3.46)$ & $2.06(1.27,3.35)$ \\
\hline $\begin{array}{l}\text { Perceived Stress Score* } \\
\text { (for every } 5 \text { unit change) }\end{array}$ & $1.33(1.21,1.46)$ & $1.32(1.19,1.46)$ \\
\hline Husband's Age* & $1.0(0.98,1.02)$ & NS \\
\hline $\begin{array}{l}\text { Condom use around the time of } \\
\text { conception }\end{array}$ & $1.02(0.50,2.11)$ & NS \\
\hline Height* (centimeters) & $1.01(.98,1.05)$ & NS \\
\hline Weight*(kilograms) & $1.02(1.01,1.04)$ & NS \\
\hline BMI & $1.05(1.00,1.11)$ & NS \\
\hline $\begin{array}{l}\text { Sex of Baby } \\
\text { Male } \\
\text { Female }\end{array}$ & $1.15(0.74,1.78) 1$ & NS \\
\hline \multicolumn{3}{|l|}{ Monthly Household Income } \\
\hline$>10000$ & $0.35(0.16$ " 0.76) & NS \\
\hline 4001 " 10000 & $0.54(0.26 " 1.13)$ & NS \\
\hline$<4000$ & 1 & \\
\hline
\end{tabular}

change of paternity because of their low prevalence of these exposures in our population. The risk factors of preeclampsia may be different in early vs. late onset of preeclampsia but we did not record the gestational age of $\mathrm{PE}$ diagnosis and therefore we were unable to categorize $\mathrm{PE}$ in early vs. late onset.

The study was conducted in accordance with the American College of Obstetricians \& Gynecologists (ACOG) criteria and ICD 9\& 10 for preeclampsia. The American College of Obstetricians \& Gynecologists (ACOG) definition for preeclampsia was used to avoid misclassification of preeclampsia with gestational hypertension as well as superimposed preeclampsia [34]. The participation rate for both control and cases subjects was $100 \%$, so there was no selection bias. The data collected was mainly by classified doctors in gynecological and Obstetrics Departments with rich clinical as well as research experience and therefore, the quality of data collected was good and accurate.
Some of the predictors like Diabetes and GDM are modifiable and preventable and others like family history of preeclampsia even if not amenable to change but still are useful in identification of women at high risk who require extra vigilance. If greater awareness of the associated risk factors leads to earlier diagnosis and improved management, there may be scope for reducing a proportion of the morbidity and mortality from preeclampsia.

\section{Conclusion}

Factors like gestational diabetes, pregestational diabetes, family history of hypertension and mental stress during pregnancy can be used as screening tools for preeclampsia prediction. These factors can help identify pregnant women who need closer monitoring for the signs of preeclampsia during early pregnancy. Every woman during the antenatal visit should also be asked for the family history hypertension in order to better estimate the possible risk of developing preeclampsia. These risk factors 
should be considered for the designs of future studies of preeclampsia in our population of Pakistani women.

\section{Competing interests}

The authors declare that they have no competing interests.

\section{Authors' contributions}

US was the PI of the study who developed the protocol as a part of her Master's thesis. She developed the design of the study, carried out the statistical analyses, participated in interpreting results, and drafted the manuscript. JH was the supervisor and she supervised the protocol, participated in interpreting results. NZ was the subject expert and gave his expertise during the study design phase. AS participated in the design of the study, coordinated the data collection in Rawalpindi hospitals and contributed to the manuscript writing. ZQ supervised the data entry and data editing along with statistical expertise. SS provided support for the protocol writing, participated in interpreting results and manuscript writing. All authors have reviewed the final manuscript.

\section{Acknowledgements}

This study was funded by University Research Council (URC), Aga Khan University Hospital, AKUH Research grant no. 051017MSC

This research was also supported by the International Maternal and Child Health Research Training Program (IMCHRT. FIC, NIH \# 5 D43 TW05497-03), Aga Khan University and University of Alabama at Birmingham (UAB).

I am thankful to Dr James Walker, (Associate professor, Department of Gynecology and Obstetrics, St James University Hospital, Leeds) for his help during protocol development through email correspondence.

\section{Author Details}

'Division of Epidemiology/Biostatistics, Department of Community Health Sciences, Aga Khan University, Karachi, Pakistan, ${ }^{2}$ Combined Military Hospital, Rawalpindi, Pakistan and ${ }^{3}$ Department of Obstetrics/Gynecology, Aga Khan University Hospital AKUH, Karachi, Pakistan

Received: 26 October 2009 Accepted: 30 April 2010 Published: 30 April 2010

\section{References}

1. Sibai B, Dekker G, Kupferminc M: Pre-eclampsia. Lancet 2005, 365(9461):785-99.

2. Landau R, Irion O: Recent data on the physiopathology of preeclampsia and recommendations for treatment. Rev Med Suisse 2005, 1(4):290-25.

3. Mostello D, Catlin TK, Roman L, Holcombe WL Jr., Leet T: Preeclampsia in the parous woman: who is at risk? Am J Obstet Gynecol 2002, 187(2):425-9.

4. Ventura SJ, Martin JA, Menacker F, Hamilton BE: Births: final data for 1999. 2001.

5. Pakistan Demographic and health survey 2006-07. National Institute of Population Studies, Islamabad, Pakistan; 2008.

6. Duckitt K, Harrington D: Risk factors for pre-eclampsia at antenatal booking: systematic review of controlled studies. Bmj 2005, 330(7491):565

7. Cohen S, Kamarck T, Mermelstein R: A global measure of perceived stress. Journal of Health and Social Behavior 1983, 24:386-96.

8. Berkowitz KM: Insulin resistance and preeclampsia. Clin Perinatol 1998, 25(4):873-85.

9. Ostlund I, Haglund B, Hanson U: Gestational diabetes and preeclampsia. Eur J Obstet Gynecol Reprod Biol 2004, 113(1):12-6.

10. England LJ, Levine RJ, Qian C, Soule LM, Schisterman EF, Yu KF: Glucose tolerance and risk of gestational diabetes mellitus in nulliparous women who smoke during pregnancy. Am J Epidemiol 2004, 160(12):1205-13.

11. Hiilesmaa V, Suhonen L, Teramo K: Glycaemic control is associated with pre-eclampsia but not with pregnancy-induced hypertension in women with type I diabetes mellitus. Diabetologia 2000, 43(12):1534-9.

12. Starcevic V, Djelmis J: Glycemic control and the risk of preeclampsia in women with gestational diabetes mellitus. Acta Med Croatica 2004, 58(5):367-71.

13. Yogev $Y$, Xenakis EM, Langer $O$ : The association between preeclampsia and the severity of gestational diabetes: the impact of glycemic control. Am J Obstet Gynecol 2004, 191(5):1655-60.
14. Drobny J: Metabolic syndrome and the risk of preeclampsia. Bratis/Lek Listy 2009, 110(7):401-3.

15. Sun $Y$, Yang $H$, Sun WJ: Risk factors for pre-eclampsia in pregnant Chinese women with abnormal glucose metabolism. Int J Gynaecol Obstet 2008, 101(1):74-6.

16. Barden A, Singh R, Walters BN, Ritchie J, Roberman B, Beilin LJ: Factors predisposing to pre-eclampsia in women with gestational diabetes. $J$ Hypertens 2004, 22(12):2371-8.

17. Kilpatrick DC: Preeclampsia and diabetes. Am J Obstet Gynecol 1997, 177(1):243.

18. Siddiqi T, Rosen B, Mimouni F, Khoury J: Hypertension during pregnancy in insulin-dependant diabetic women. Obstet Gynecol 1991, 77:514-9.

19. Sibai BM, Caritis S, Hauth J, Lindheimer M, VanDorsten JP, MacPherson C: Risks of preeclampsia and adverse neonatal outcomes among women with pregestational diabetes mellitus. In Am J Obstet Gynecol Volume 182. Issue 2 National Institute of Child Health and Human Development Network of Maternal-Fetal Medicine Units; 2000:364-9.

20. Sibai BM: Risk factors, pregnancy complications, and prevention of hypertensive disorders in women with pregravid diabetes mellitus. J Matern Fetal Med 2000, 9(1):62-5.

21. Reaven GM, Lithell H, Landsberg L: Hypertension and associated metabolic abnormalities--the role of insulin resistance and the sympathoadrenal system. N Engl J Med 1996, 334(6):74-81.

22. Rowe JW, Young JB, Minaker KL, Stevens AL, Pallotta J, Landsberg L: Effect of insulin and glucose infusions on sympathetic nervous system activity in normal man. Diabetes 1 1981, 30(3):219-25.

23. Ness RB, Markovic N, Bass D, Harger G, Roberts JM: Family history of hypertension, heart disease, and stroke among women who develop hypertension in pregnancy. Obstet Gynecol 2003, 102(6):1366-71.

24. Sanchez SE, Zhang C, Qiu CF, Williams MA: Family history of hypertension and diabetes in relation to preeclampsia risk in Peruvian women. Gynecol Obstet Invest 2003, 56(3):128-32.

25. Qiu C, Williams MA, Leisenring WM, Sorensen TK, Frederick IO, Dempsey JC, Luthy DA: Family History of Hypertension and Type 2 Diabetes in Relation to Preeclampsia Risk. Hypertension 2003, 41:408-13.

26. Mahomed K, Williams MA, Woelk GB, Jenkins-Woelk L, Mudzamiri S, Longstaff $L$ : Risk factors for pre-eclampsia among Zimbabwean women: maternal arm circumference and other anthropometric measures of obesity. Paediatr Perinat Epidemiol 1998, 12(3):253-62.

27. Merviel P, Touzart L, Deslandes V, Delmas M, Coicaud M, Gondry J: Risk factors of preeclampsia in single pregnancy. J Gynecol Obstet Biol Reprod (Paris) 2008, 37(5):477-82.

28. Kurki T, Hiilesmaa V, Raitasalo R, Mattila H, Ylikorkala O: Depression and anxiety in early pregnancy and risk for preeclampsia. Obstet Gynecol 2000, 95(4):487-90.

29. Qiu C, Williams M A, Calderon-Margalit R, Cripe S M, Sorensen T K: Preeclampsia Risk in Relation to Maternal Mood and Anxiety Disorders Diagnosed Before or During Early Pregnancy. American Journal of Hypertension 2009, 22(4):397-402.

30. Landsbergis PA, Hatch MC: Psychosocial work stress and pregnancyinduced hypertension. Epidemiology 1996, 7(4):346-51.

31. Klonoff-Cohen HS, Cross JL, Pieper CF: Job stress and preeclampsia. Epidemiology 1996, 7(3):245-9.

32. Sullivan P, Schoentgen S, DeQuattro V, Procci W, Levine D, Meulen J Van der, Bornheimer J: Anxiety, anger, and neurogenic tone at rest and in stress in patients with primary hypertension. Hypertension 1981, 6(Pt 2):119-123.

33. Potter WZ, Manji HK: Catecholamines in depression: an update. Clin Chem 1994, 40(2):279-87.

34. Schroeder BM: ACOG practice bulletin on diagnosing and managing preeclampsia and eclampsia. American College of Obstetricians and Gynecologists. Am Fam Physician 2002, 66(2):330-1.

Pre-publication history

The pre-publication history for this paper can be accessed here: http://www.biomedcentral.com/1472-6874/10/14/prepub

doi: $10.1186 / 1472-6874-10-14$

Cite this article as: Shamsi et al., A multicentre matched case control study of risk factors for Preeclampsia in healthy women in Pakistan BMC Women's Health 2010, 10:14 